\title{
MEASURE HOMOLOGY
}

\author{
SØREN KOLD HANSEN
}

\begin{abstract}
.
Let $X$ be a topological space, $\operatorname{Sin}_{k}(X)$ the space of singular $k$-simplices with the compact-open topology, and let $\mathscr{C}_{k}(X)$ be the real vector space of all compactly supported signed Borel Measures of bounded total variation on $\operatorname{Sin}_{k}(X)$. There are linear operators $\partial: \mathscr{C}_{k}(X) \rightarrow \mathscr{C}_{k-1}(X)$, so that $\left\{\mathscr{C}_{*}(X), \partial\right\}$ is a chain complex. The homology $H_{*}^{\mu}(X)$ is the measure homology of $X$ of Thurston and Gromov. The main results in this paper are that $H_{*}^{\mu}(-)$ satisfies the EilenbergSteenrod axioms for a wide class of topological spaces including all metric spaces, and is ordinary homology with real coefficients for $\mathrm{CW}$-complexes.
\end{abstract}

\section{Introduction.}

Measure homology was introduced by Gromov and Thurston in [T] $\S 6$ in connection with Gromov's theorem that the Gromov norm of a closed oriented hyperbolic $n$-manifold $M$ equals the volume of $M$ divided by the supremum of the volumes of the geodesic $n$-simplices in the hyperbolic $n$ space.

For a measurable space $(X, \wp)$, let $\mathscr{V}(X, \wp)$ be the vector space of all signed measures of bounded total varation. The total variation of a signed measure $\mu$ on $(X, \wp)$ is $\|\mu\|=\mu^{+}(X)-\mu^{-}(X)$ where $\mu=\mu^{+}-\mu^{-}$is the Jordan decomposition of $\mu$ into its positive and negative variation. A measure $\mu$ on $(X, \wp)$ has support in $A \in \wp, \operatorname{Supp}(\mu) \subseteq A$, if $\mu(A \cap B)=\mu(B)$ for all $B \in \wp$. We write $\mathscr{B}(X)$ for the Borel $\sigma$-algebra on the space $X$, and define a linear subspace of $\mathscr{V}(X, \mathscr{B}(X))$ by

$$
\mathscr{M}_{c}(X)=\{\mu \in \mathscr{V}(X, \mathscr{B}(X)) \mid \mu \text { has compact support }\} .
$$

A continuous map $f: X \rightarrow Y$ induces a linear map $f_{*}: \mathscr{M}_{c}(X) \rightarrow \mathscr{M}_{c}(Y)$, namely the image measure of $\mu$ under $f$.

Let $\operatorname{Sin}_{k}(X)$ be the set of continuous maps from the standard $k$-simplex $\Delta^{k}$ to the space $X$ with the compact-open topology, and set

$$
\mathscr{C}_{k}(X)=\mathscr{M}_{c}\left(\operatorname{Sin}_{k}(X)\right) \text {. }
$$

Received July 2, 1996. 
The $i$ th face map $\delta^{i}: \Delta^{k-1} \rightarrow \Delta^{k}$ induces a continuous map $\partial_{i}: \operatorname{Sin}_{k}(X) \rightarrow \operatorname{Sin}_{k-1}(X), \quad \partial_{i}(\sigma)=\sigma \circ \delta_{i}, \quad$ and hence a linear map $\left(\partial_{i}\right)_{*}: \mathscr{C}_{k}(X) \rightarrow \mathscr{C}_{k-1}(X)$. The measure chain complex is the spaces $\mathscr{C}_{k}(X)$ together with the boundary operators $\partial=\sum_{i=0}^{k}(-1)^{i}\left(\partial_{i}\right)_{*}$. The homology of $\mathscr{C}_{*}(X)$ is denoted $H_{*}^{\mu}(X)$ and is the measure homology of $X$, cf. [T]. Actually, in [T], the authors only defined $\mathscr{C}_{*}(X)$ when $X$ is a smooth manifold and used the sets $\operatorname{Sin}_{k}^{1}(X)$ of singular $k$-simplices of class $C^{1}$ with the $C^{1}$ topology instead of $\operatorname{Sin}_{k}(X)$. We shall see that this makes no difference. The main theorem of this paper is the following result, listed without proof in the case of smooth manifolds in $[T] \S 6$ p. (6.7):

THEOREM 1.1. The measure homology functor satisfies the EilenbergSteenrod axioms on the category of metric spaces.

Remarks. 1) Actually we prove that $H_{*}^{\mu}(X)$ satisfies the Eilenberg-Steenrod axioms for all Hausdorff spaces $X$ such that $\operatorname{Sin}_{k}(X)$ and $\operatorname{Sin}_{k}(A)$ are normal for all $k \geq 0$ and all $A \subseteq X$. This is indeed satisfied if $X$ is a metric space. Note that normality of $X$ does not imply normality of $\operatorname{Sin}_{k}(X)$. Actually A. H. Stone showed in [S] that if $I=[0,1]$ and $Y$ is the product of uncountably many copies of $I$ then $Y^{I}$ is not normal, where $Y^{I}$ is the space of maps of $I$ into $Y$ with the compact-open topology.

2) If $X$ is a smooth manifold theorem 1.1 and the proof we give for it is still valid if one uses the sets $\operatorname{Sin}_{k}^{r}(X)$ of singular $k$-simplices of class $C^{r}$ with the $C^{r}$ topology instead of $\operatorname{Sin}_{k}(X)=\operatorname{Sin}_{k}^{0}(X)$ to define measure homology, $1 \leq r \leq \infty$.

I would here like to thank H. J. Munkholm for drawing my attention to this problem and I. Madsen and J. Tornehave for guidance.

\section{The measure homology functor.}

In the preceding section we introduced the measure chain complex $\mathscr{C}_{*}(X)$ for an arbitrary topological space $X$. A map $f: X \rightarrow Y$ induces linear maps $\bar{f}: \mathscr{C}_{k}(X) \rightarrow \mathscr{C}_{k}(Y)$ by $\bar{f}=\left(f_{\#}\right)_{*}$ where $f_{\#}: \operatorname{Sin}_{k}(X) \rightarrow \operatorname{Sin}_{k}(Y)$ is as usual. Instead of $\bar{f}$ we usually write $f: \mathscr{C}_{k}(X) \rightarrow \mathscr{C}_{k}(Y)$. This makes $\mathscr{C}_{*}(-)$ a covariant functor and turns $H_{*}^{\mu}(-)$ into a covariant functor in a standard way.

One can generalize the above to pairs of Hausdorff spaces $(X, A)$. We have $\mathscr{B}(A)=\{Z \cap A \mid Z \in \mathscr{B}(X)\}$, so that $\mathscr{B}(A)=\{Z \in \mathscr{B}(X) \mid Z \subseteq A\} \subseteq \mathscr{B}(X)$ if $A \in \mathscr{B}(X)$. For an arbitrary set $E, \mathscr{P}(E)=\{A \mid A \subseteq E\}$ denotes the power set of $E$. Taking direct images, $f: E \rightarrow F$ induces $\mathscr{P}(f): \mathscr{P}(E) \rightarrow \mathscr{P}(F)$ and makes $\mathscr{P}(-)$ a covariant functor. For a homeomorphism $f: X \rightarrow Y$, $\left.\mathscr{P}(f)\right|_{\mathscr{B}(X)}: \mathscr{B}(X) \rightarrow \mathscr{B}(Y)$ is a bijection. We use below that $\operatorname{Sin}_{k}(X)$ is a Hausdorff space if and only if $X$ is. 
Lemma 2.1. For $(X, A)$ a pair of Hausdorff spaces, the inclusion $i: A \rightarrow X$ induces a monomorphism $i: \mathscr{C}_{*}(A) \rightarrow \mathscr{C}_{*}(X)$.

Proof. If $K$ is a compact subset of $\operatorname{Sin}_{k}(A)$ then $L=i_{\#}(K)$ is a compact subset of $\operatorname{Sin}_{k}(X)$ and $\left.i_{\#}\right|_{K}: K \rightarrow L$ is a homeomorphism. Let $\mu_{1}, \mu_{2} \in \mathscr{C}_{k}(A)$ with compact supports $K_{1}, K_{2}$, and $i\left(\mu_{1}\right)=i\left(\mu_{2}\right)$. If $B \in \mathscr{B}\left(\operatorname{Sin}_{k}(A)\right)$ then $B \cap\left(K_{2} \backslash K_{1}\right)=K_{2} \cap\left(B \backslash K_{1}\right) \in \mathscr{B}\left(K_{2}\right)$, so

$$
\begin{aligned}
\mu_{2}\left(B \cap\left(K_{2} \backslash K_{1}\right)\right) & =i\left(\mu_{2}\right)\left(i_{\#}\left(B \cap\left(K_{2} \backslash K_{1}\right)\right)\right)=i\left(\mu_{1}\right)\left(i_{\#}\left(B \cap\left(K_{2} \backslash K_{1}\right)\right)\right) \\
& =\mu_{1}\left(B \cap\left(K_{2} \backslash K_{1}\right)\right)=0 .
\end{aligned}
$$

Thus $K_{1} \cap K_{2}$ is a support for $\mu_{2}$, and, symmetrically for $\mu_{1}$. If $B \in \mathscr{B}\left(\operatorname{Sin}_{k}(X)\right)$,

$$
\mu_{\nu}\left(i_{\#}^{-1}(B)\right)=\mu_{\nu}\left(i_{\#}^{-1}(B) \cap K_{1} \cap K_{2}\right)=\mu_{\nu}\left(i_{\#}^{-1}\left(B \cap L_{1} \cap L_{2}\right)\right)
$$

where $L_{\nu}=i_{\#}\left(K_{\nu}\right), \nu=1,2$. But

$$
\begin{aligned}
\mathscr{B}\left(K_{1} \cap K_{2}\right) & =\left\{i_{\#}^{-1}(D) \mid D \in \mathscr{B}\left(L_{1} \cap L_{2}\right)\right\} \\
& =\left\{i_{\#}^{-1}\left(B \cap L_{1} \cap L_{2}\right) \mid B \in \mathscr{B}\left(\operatorname{Sin}_{k}(X)\right)\right\}
\end{aligned}
$$

so $\mu_{1}=\mu_{2}$ on $\mathscr{B}\left(K_{1} \cap K_{2}\right)$ hence on all of $\mathscr{B}\left(\operatorname{Sin}_{k}(A)\right.$.

We let $\mathscr{C}_{*}(X, A)$ be the cokernel of $i: \mathscr{C}_{*}(A) \rightarrow \mathscr{C}_{*}(X)$, so that we have an exact sequence

$$
0 \longrightarrow \mathscr{C}_{*}(A) \stackrel{i}{\longrightarrow} \mathscr{C}_{*}(X) \stackrel{\pi}{\longrightarrow} \mathscr{C}_{*}(X, A) \longrightarrow 0
$$

of chain complexes. The homology groups of $\mathscr{C}_{*}(X, A)$ are the relative measure homology groups of $(X, A)$ and are denoted $H_{*}^{\mu}(X, A)$. A map $f:(X, A) \rightarrow(Y, B)$ of pairs of Hausdorff spaces induces a commutative diagram

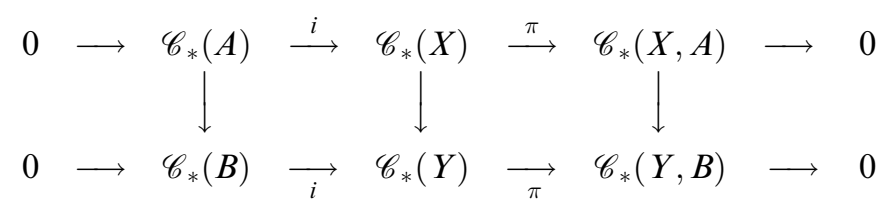

of chain maps. Thus we get as usual a long exact homology sequence, natural in $(X, A)$ :

$$
\cdots \stackrel{\partial_{*}}{\longrightarrow} H_{k}^{\mu}(A) \stackrel{i_{*}}{\longrightarrow} H_{k}^{\mu}(X) \stackrel{j_{*}}{\longrightarrow} H_{k}^{\mu}(X, A) \stackrel{\partial_{*}}{\longrightarrow} H_{k-1}^{\mu}(A) \stackrel{i_{*}}{\longrightarrow} \cdots
$$




\section{Proof of theorem 1.1.}

In this section we verify the homotopy axiom, excision and the dimension axiom, i.e.

i) If $f_{0}, f_{1}:(X, A) \rightarrow(Y, B)$ are homotopic as maps of pairs of Hausdorff spaces, then $H_{*}^{\mu}\left(f_{0}\right)=H_{*}^{\mu}\left(f_{1}\right): H_{*}^{\mu}(X, A) \rightarrow H_{*}^{\mu}(Y, B)$.

ii) If $(X, A)$ is a pair of metric spaces and $U \subseteq A$ has $\bar{U} \subseteq \operatorname{Int}(A)$, then the inclusion map $i:(X-U, A-U) \rightarrow(X, A)$ induces an isomorphism on homology.

iii) If $X$ is a one-point space, then $H_{k}^{\mu}(X)=0$ for $k \neq 0$ and $H_{0}^{\mu}(X)=\mathrm{R}$.

If one works with complex measures instead of real measures the only difference is that $H_{0}^{\mu}(X)=\mathrm{C}$ for a one-point space $X$. We start by showing the easy i) and iii).

Proof of iii). Since $\operatorname{Sin}_{k}(X)$ has only one element $\varphi_{k}, \mathscr{B}\left(\operatorname{Sin}_{k}(X)\right)=$ $\left\{\emptyset,\left\{\varphi_{k}\right\}\right\}$ and $\mu \in \mathscr{C}_{k}(X)$ is completely determined by the value $\mu\left(\left\{\varphi_{k}\right\}\right)$. If $r \in \mathrm{R}$ we get an element $\mu_{r}^{k} \in \mathscr{C}_{k}(X)$ defined by $\mu_{r}^{k}(\emptyset)=0, \mu_{r}^{k}\left(\left\{\varphi_{k}\right\}\right)=r$. This shows that $\mathscr{C}_{k}(X) \cong \mathrm{R}$, and a simple calculation shows that

$$
\partial\left(\mu_{r}^{k}\right)= \begin{cases}0 & , k \text { odd } \\ \mu_{r}^{k-1} & , k \text { even and } k>0 .\end{cases}
$$

Since $\partial \mu=0$ for all $\mu \in \mathscr{C}_{0}(X)$ by definition the result follows.

Proof of i). We just do the absolute case, $A=B=\emptyset$. Let $\lambda_{t}: X \rightarrow X \times I$ be given by $\lambda_{t}(x)=(x, t), I=[0,1]$ and let $F: X \times I \rightarrow Y$ be a homotopy between $f_{0}$ and $f_{1}$. Then $F \lambda_{0}=f_{0}$ and $F \lambda_{1}=f_{1}$ and it suffices to show that $H_{*}^{\mu}\left(\lambda_{0}\right)=H_{*}^{\mu}\left(\lambda_{1}\right): H_{*}^{\mu}(X) \rightarrow H_{*}^{\mu}(X \times I)$. To show this we construct a chain homotopy $P: \mathscr{C}_{*}(X) \rightarrow \mathscr{C}_{*}(X \times I)$ between $\lambda_{0}$ and $\lambda_{1}$. For $i=0,1, \ldots, k$ we define maps $Q_{i}: \operatorname{Sin}_{k}(X) \rightarrow \operatorname{Sin}_{k+1}(X \times I)$ by

$$
Q_{i}(\sigma)\left(t_{0}, \ldots, t_{k+1}\right)=\sigma\left(t_{0}, \ldots, t_{i-1}, t_{i}+t_{i+1}, t_{i+2}, \ldots, t_{k+1}\right) \times\left(1-\sum_{l=0}^{i} t_{l}\right)
$$

for $\sigma \in \operatorname{Sin}_{k}(X)$ and $\left(t_{0}, \ldots, t_{k+1}\right) \in \Delta^{k+1}$. The $Q_{i}$ are continuous and induce linear maps $\left(Q_{i}\right)_{*}: \mathscr{C}_{k}(X) \rightarrow \mathscr{C}_{k+1}(X \times I)$. Define $P_{k}: \mathscr{C}_{k}(X) \rightarrow \mathscr{C}_{k+1}(X \times I)$ by $P_{k}=\sum_{i=0}^{k}(-1)^{i}\left(Q_{i}\right)_{*}$. A tedious calculation shows that the $P_{k}$ form a natural chain homotopy between $\lambda_{0}$ and $\lambda_{1}$. The general case now follows in a standard way, by using naturality of $P$.

We now begin the proof of ii). Let $U$ be an open subset of $X$ and $i: U \rightarrow X$ the inclusion map. Then $V=i_{\#}\left(\operatorname{Sin}_{k}(U)\right)$ is open in $\operatorname{Sin}_{k}(X)$ and $i_{\#}: \operatorname{Sin}_{k}(U) \rightarrow V$ is a homeomorphism. It follows that $\mathscr{P}\left(i_{\#}\right)$ : $\mathscr{B}\left(\operatorname{Sin}_{k}(U)\right) \rightarrow \mathscr{B}(V)$ is a bijection so that 


$$
\mathscr{B}\left(\operatorname{Sin}_{k}(U)\right)=\left\{i_{\#}^{-1}(B) \mid B \in \mathscr{B}(V)\right\}=\left\{i_{\#}^{-1}(B) \mid B \in \mathscr{B}\left(\operatorname{Sin}_{k}(X)\right)\right\} .
$$

For a family $\mathscr{U}=\left\{U_{\alpha} \mid \alpha \in I\right\}$ of (not necessarily open) subsets of $X$ we consider the subchain complex of $\mathscr{C}_{*}(X)$ of " $\mathscr{U}$-small" measures

$$
\mathscr{C}_{k}^{\mathscr{U}}(X)=\sum_{\alpha \in I} \mathscr{C}_{k}^{U_{\alpha}}(X), \quad \mathscr{C}_{k}^{U_{\alpha}}(X)=i_{\alpha}\left(\mathscr{C}_{k}\left(U_{\alpha}\right)\right)
$$

For the family $\mathscr{U}$ we let $\operatorname{Int}(\mathscr{U})$ be the collection of interiors of elements of $\mathscr{U}$. We then have

THEOREM 3.1. Let $X$ be a metric space and let $\mathscr{U}$ be a family of subsets of $X$ such that Int $(\mathscr{U})$ is a covering of $X$. Then the inclusion chain map $I: \mathscr{C}_{*}^{\mathscr{U}}(X) \rightarrow \mathscr{C}_{*}(X)$ induces an isomorphism on homology.

The proof for this theorem is deferred to $\S 4$. As in the case of singular homolog the excision axiom follows at once. Let $\mathscr{U}=\left\{U_{\alpha} \mid \alpha \in I\right\}$ and $X$ be as in theorem 3.1 and let $\mathscr{U} \cap A=\left\{U_{\alpha} \cap A \mid \alpha \in I\right\}$. The commutative diagram of inclusion maps

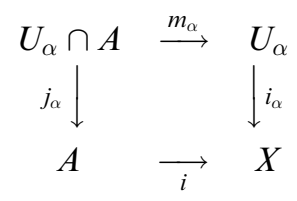

shows that $i\left(j_{\alpha}\left(\mathscr{C}_{k}\left(U_{\alpha} \cap A\right)\right)\right) \subseteq i_{\alpha}\left(\mathscr{C}_{k}\left(U_{\alpha}\right)\right)$ so $i\left(\mathscr{C}_{*}^{\mathscr{U} \cap A}(X)\right)$ is a subcomplex of $\mathscr{C}_{*}^{\mathscr{U}}(X)$. Setting $\mathscr{C}_{*}(\mathscr{U}, \mathscr{U} \cap A)=\mathscr{C}_{*}^{\mathscr{U}}(X) / i\left(\mathscr{C}_{*}^{\mathscr{U} \cap A}(A)\right)$, we have a commutative diagram of chain maps

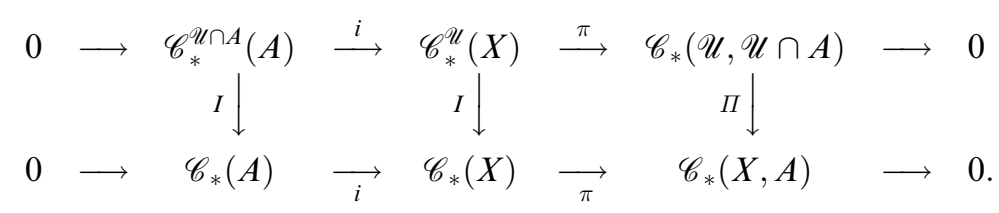

By the preceding theorem the inclusions $I$ induce isomorphisms on homology, and the five-lemma yields that $\Pi$ induces an isomorphism on homo$\log$.

Proof of ii). Let $\mathscr{U}=\{X-U, \operatorname{Int}(A)\}$. Then $\mathscr{U} \cap A=\{A-U, \operatorname{Int}(A)\}$ and we have that $\operatorname{Int}(\mathscr{U})$ and $\operatorname{Int}(\mathscr{U} \cap A)$ cover respectively $X$ and $A$. Now let

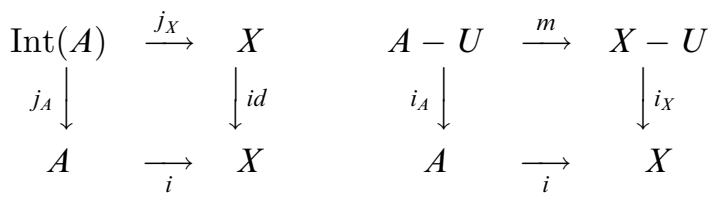


be commutative diagrams of inclusion maps. Then we have

$$
\begin{aligned}
\mathscr{C}_{k}^{\mathscr{U} A}(A) & =i_{A}\left(\mathscr{C}_{k}(A-U)\right)+j_{A}\left(\mathscr{C}_{k}(\operatorname{Int}(A))\right) \\
\mathscr{C}_{k}^{\mathscr{U}}(X) & =i_{X}\left(\mathscr{C}_{k}(X-U)\right)+j_{X}\left(\mathscr{C}_{k}(\operatorname{Int}(A))\right)
\end{aligned}
$$

and therefore $i\left(\mathscr{C}_{k}^{\mathscr{U} \cap A}(A)\right)=i_{X} \circ m\left(\mathscr{C}_{k}(A-U)\right)+j_{X}\left(\mathscr{C}_{k}(\operatorname{Int}(A))\right)$. This implies the isomorphisms

$$
\begin{aligned}
\mathscr{C}_{*}(\mathscr{U}, \mathscr{U} \cap A) & =\mathscr{C}_{*}^{\mathscr{U}}(X) / i\left(\mathscr{C}_{*}^{\mathscr{U} \cap A}(A)\right) \cong i_{X}\left(\mathscr{C}_{*}(X-U)\right) / i_{X}\left(m\left(\mathscr{C}_{*}(A-U)\right)\right) \\
& \cong \mathscr{C}_{*}(X-U) / m\left(\mathscr{C}_{*}(A-U)\right)=\mathscr{C}_{*}(X-U, A-U)
\end{aligned}
$$

The first isomorphism follows by the fact that

$$
\left[i_{X} \circ m\left(\mathscr{C}_{k}(A-U)\right)+j_{X}\left(\mathscr{C}_{k}(\operatorname{Int}(A))\right] \cap i_{X}\left(\mathscr{C}_{k}(X-U)\right)=i_{X} \circ m\left(\mathscr{C}_{k}(A-U)\right)\right.
$$

Since $\Pi: \mathscr{C}_{*}(\mathscr{U}, \mathscr{U} \cap A) \rightarrow \mathscr{C}_{*}(X, A)$ induces an isomorphism on homology the result follows.

\section{Proof of theorem 3.1.}

The proof of theorem 3.1 uses the standard ideas from barycentric subdivision in singular theory, which we begin by recalling, cf. [D]. The subdivision homomorphisms $\beta_{q}: S_{q}(X) \rightarrow S_{q}(X), q \in \mathrm{Z}$, are inductively defined in the following way:

Let $\imath_{q} \in S_{q}\left(\Delta^{q}\right)$ denotes the identity map of the standard $q$-simplex $\Delta^{q}$ with vertices the standard basis $\left\{e_{i}\right\}$ in $\mathrm{R}^{q+1}$ and let $B_{q}=\sum_{i=0}^{q} \frac{1}{q+1} e_{i}$ be the barycenter of $\Delta^{q}$. Write $B_{q}$. for the cone construction (see [D] chap. III (4.7) p. 34), and set

$$
\beta_{0}=\mathrm{id} \quad \beta_{q}\left(\imath_{q}\right)=B_{q} \cdot \beta_{q-1}\left(\partial \imath_{q}\right), \quad q>0 .
$$

One defines $\beta_{q}: \operatorname{Sin}_{q}(X) \rightarrow S_{q}(X)$ by $\beta_{q}(\sigma)=\sigma_{\#}\left(\beta_{q}\left(\imath_{q}\right)\right)$. Then

$$
\beta: S_{*}(X) \rightarrow S_{*}(X)
$$

is a natural chain map, [D] p. 41. For later use we need to explicate the natural chain homotopy $s: \beta \simeq \operatorname{id}_{S_{*}(X)}$, [D] p. 42. It is 0 for $q=0$ and is given by

$$
s_{q}\left(\imath_{q}\right)=B_{q} \cdot\left(\beta_{q}\left(\imath_{q}\right)-\imath_{q}-s_{q-1}\left(\partial \imath_{q}\right)\right) \in S_{q+1}\left(\Delta^{q}\right)
$$

on $\imath_{q} \in S_{q}\left(\Delta^{q}\right)$ for $q>0$. For a general $\sigma \in \operatorname{Sin}_{q}(X), s_{q}(\sigma)=\sigma_{\#}\left(s_{q}\left(\imath_{q}\right)\right)$.

We now want to define a "subdivision" homomorphism $\beta: \mathscr{C}_{*}(X) \rightarrow$ $\mathscr{C}_{*}(X)$ for the measure theoretical chain complex and a chain homotopy $s: \beta \simeq \operatorname{id}_{\mathscr{C}_{*}(X)}$. To this end we write out the construction in (1) in a form $\beta_{q}=\sum_{\nu \in A_{q}} r_{\nu} \beta_{q}^{\nu}$ where $\beta_{q}^{\nu}: \operatorname{Sin}_{q}(X) \rightarrow \operatorname{Sin}_{q}(X)$ are continuous and induce 
linear maps $\left(\beta_{q}^{\nu}\right)_{*}: \mathscr{C}_{q}(X) \rightarrow \mathscr{C}_{q}(X)$. Thus we can define our measure theoretical "subdivision" homomorphism by $\beta_{q}=\sum_{\nu \in A_{q}} r_{\nu}\left(\beta_{q}^{\nu}\right)_{*}$. This procedure will also be used to define the chain homotopy $s: \beta \simeq i d_{\mathscr{C}_{*}(X)}$. For $q \geq 1$ the explicit formula is

$$
\beta_{q}\left(\imath_{q}\right)=\sum_{k_{q}=0}^{q} \sum_{k_{q-1}=0}^{q-1} \cdots \sum_{k_{1}=0}^{1}(-1)^{k_{1}+\cdots+k_{q}} \sigma_{q}^{k_{1} \ldots k_{q}}
$$

where

$$
\sigma_{q}^{k_{1} \ldots k_{q}}=B_{q} \cdot\left(\delta^{k_{q}} \circ\left(B_{q-1} \cdot\left(\delta^{k_{q-1}} \circ \cdots B_{2} \cdot\left(\delta^{k_{2}} \circ\left(B_{1} \cdot \delta^{k_{1}}\right)\right) \cdots\right)\right)\right) \in \operatorname{Sin}_{q}\left(\Delta^{q}\right) .
$$

Thus we get maps $\beta_{q}: \operatorname{Sin}_{q}(X) \rightarrow S_{q}(X)$,

$$
\beta_{q}=\sum_{k_{q}=0}^{q} \sum_{k_{q-1}=0}^{q-1} \cdots \sum_{k_{1}=0}^{1}(-1)^{k_{1}+\cdots+k_{q}} \beta_{q}^{k_{1} \ldots k_{q}}
$$

where $\beta_{q}^{k_{1} \ldots k_{q}}(\sigma)=\sigma \circ \sigma_{q}^{k_{1} \ldots k_{q}}$. Similarly when $q \geq 1$ we have

$$
\begin{aligned}
s_{q}\left(\imath_{q}\right) & =\sum_{\nu=1}^{q}(-1)^{q-\nu} \sum_{k_{q}=0}^{q} \sum_{k_{q-1}=0}^{q-1} \ldots \sum_{k_{1}=0}^{1}(-1)^{k_{1}+\cdots+k_{q}} f_{q, \nu}\left(k_{1}, \ldots, k_{q}\right) \\
& -\sum_{\nu=1}^{q-1}(-1)^{q-\nu} \sum_{k_{q}=0}^{q} \sum_{k_{q-1}=0}^{q-1} \ldots \sum_{k_{\nu+1}=0}^{\nu+1}(-1)^{k_{\nu+1}+\cdots+k_{q}} g_{q, \nu}\left(k_{\nu+1}, \ldots, k_{q}\right)-g_{q, q}
\end{aligned}
$$

where

$$
\begin{aligned}
& f_{q, \nu}\left(k_{1}, \ldots, k_{q}\right)=B_{q} \cdot\left(\delta^{k_{q}} \circ\left(B_{q-1} \cdot\left(\delta^{k_{q-1}} \circ \cdots\left(\delta^{k_{\nu+1}} \circ\left(B_{\nu} \cdot \sigma_{\nu}^{k_{1} \cdots k_{\nu}}\right)\right) \cdots\right)\right)\right) \\
& g_{q, \nu}\left(k_{\nu+1}, \ldots, k_{q}\right)=B_{q} \cdot\left(\delta^{k_{q}} \circ\left(B_{q-1} \cdot\left(\delta^{k_{q-1}} \circ \cdots\left(\delta^{k_{\nu+1}} \circ\left(B_{\nu} \cdot \imath_{\nu}\right)\right) \cdots\right)\right),\right. \\
& \nu=1, \ldots, q-1, \text { and }
\end{aligned}
$$

$$
\begin{aligned}
& f_{q, q}\left(k_{1}, \ldots, k_{q}\right)=B_{q} \cdot \sigma_{q}^{k_{1} \cdots k_{q}} \\
& g_{q, q}=B_{q} \cdot \imath_{q}
\end{aligned}
$$

are elements of $\operatorname{Sin}_{q+1}\left(\Delta^{q}\right)$, so $s_{q}: \operatorname{Sin}_{q}(X) \rightarrow S_{q+1}(X)$ is given by

$$
\begin{aligned}
s_{q} & =\sum_{\nu=1}^{q}(-1)^{q-\nu} \sum_{k_{q}=0}^{q} \sum_{k_{q-1}=0}^{q-1} \cdots \sum_{k_{1}=0}^{1}(-1)^{k_{1}+\cdots+k_{q}} f_{q, \nu}^{k_{1} \ldots k_{q}} \\
& -\sum_{\nu=1}^{q-1}(-1)^{q-\nu} \sum_{k_{q}=0}^{q} \sum_{k_{q-1}=0}^{q-1} \cdots \sum_{k_{\nu+1}=0}^{\nu+1}(-1)^{k_{\nu+1}+\cdots+k_{q}} g_{q, \nu}^{k_{\nu+1} \ldots k_{q}}-h_{q, q}
\end{aligned}
$$

where $f_{q, \nu}^{k_{1} \ldots k_{q}}, g_{q, \nu}^{k_{\nu+1} \ldots k_{q}}, h_{q, q}: \operatorname{Sin}_{q}(X) \rightarrow \operatorname{Sin}_{q+1}(X)$ are defined by 


$$
\begin{aligned}
& f_{q, \nu}^{k_{1} \ldots k_{q}}(\sigma)=\sigma \circ f_{q, \nu}\left(k_{1}, \ldots, k_{q}\right) \\
& g_{q, \nu}^{k_{\nu+1} \ldots k_{q}}(\sigma)=\sigma \circ g_{q, \nu}\left(k_{\nu+1}, \ldots, k_{q}\right) \\
& h_{q, q}(\sigma)=\sigma \circ q_{q, q} .
\end{aligned}
$$

In the following we shorten notation and write (3) as $\beta_{q}=\sum_{\nu \in A_{q}} r_{\nu} \beta_{q}^{\nu}$ where $A_{q}$ is the set of $q$-tuples $\left(a_{1}, \ldots, a_{q}\right), a_{i}=0,1, \ldots, i$, and $r_{\left(k_{1}, \ldots, k_{q}\right)}=(-1)^{k_{1}+\cdots+k_{q}}$. For $q \geq 2$ we then have

$$
\begin{aligned}
& \partial \beta_{q}=\sum_{j=0}^{q}(-1)^{j} \partial_{j}\left(\sum_{\nu \in A_{q}} r_{\nu} \beta_{q}^{\nu}\right)=\sum_{j=0}^{q} \sum_{\nu \in A_{q}}(-1)^{j} r_{\nu} \partial_{j} \circ \beta_{q}^{\nu} \\
& \beta_{q-1} \partial=\sum_{\alpha \in A_{q-1}} r_{\alpha} \beta_{q-1}^{\alpha}\left(\sum_{j=0}^{q}(-1)^{j} \partial_{j}\right)=\sum_{j=0}^{q} \sum_{\alpha \in A_{q-1}}(-1)^{j} r_{\alpha} \beta_{q-1}^{\alpha} \circ \partial_{j} .
\end{aligned}
$$

Now $\partial \beta_{q}=\beta_{q-1} \partial$ by (2) so in particular $\partial \beta_{q}\left(\imath_{q}\right)=\beta_{q-1} \partial\left(\imath_{q}\right)$, i.e.

$$
\sum_{j=0}^{q} \sum_{\nu \in A_{q}}(-1)^{j} r_{\nu} \sigma_{q}^{\nu} \circ \delta^{j}=\sum_{j=0}^{q} \sum_{\alpha \in A_{q-1}}(-1)^{j} r_{\alpha} \delta^{j} \circ \sigma_{q-1}^{\alpha} .
$$

Since $\operatorname{Sin}_{q-1}\left(\Delta^{q}\right)$ is a basis for $S_{q-1}\left(\Delta^{q}\right)$ we can write

$$
\begin{aligned}
& \sum_{j=0}^{q} \sum_{\nu \in A_{q}}(-1)^{j} r_{\nu} \sigma_{q}^{\nu} \circ \delta^{j}=\sum_{\lambda \in M_{q}} t_{\lambda} \tau_{q}^{\lambda} \\
& \sum_{j=0}^{q} \sum_{\alpha \in A_{q-1}}(-1)^{j} r_{\alpha} \delta^{j} \circ \sigma_{q-1}^{\alpha}=\sum_{\mu \in N_{q}} s_{\mu} \omega_{q}^{\mu},
\end{aligned}
$$

where

$$
\begin{aligned}
& \left\{\tau_{q}^{\lambda} \mid \lambda \in M_{q}\right\} \subseteq\left\{\sigma_{q}^{\nu} \circ \delta^{j} \mid(\nu, j) \in A_{q} \times\{0,1, \ldots, q\}\right\} \\
& \left\{\omega_{q}^{\mu} \mid \mu \in N_{q}\right\} \subseteq\left\{\delta^{j} \circ \sigma_{q-1}^{\alpha} \mid(j, \alpha) \in\{0,1, \ldots, q\} \times A_{q-1}\right\},
\end{aligned}
$$

and $\lambda_{1} \neq \lambda_{2} \Rightarrow \tau_{q}^{\lambda_{1}} \neq \tau_{q}^{\lambda_{2}}$ and $\mu_{1} \neq \mu_{2} \Rightarrow \omega_{q}^{\mu_{1}} \neq \omega_{q}^{\mu_{2}}$ and $t_{\lambda} \neq 0$ for all $\lambda \in M_{q}$ and $s_{\mu} \neq 0$ for all $\mu \in N_{q}$. We observe that $M_{q}$ and $N_{q}$ contain the same number of elements and that for all $\lambda \in M_{q}$ there exists a $\mu \in N_{q}$ such that $s_{\mu}=t_{\lambda}$ and $\omega_{q}^{\mu}=\tau_{q}^{\lambda}$. Now let $T_{q}^{\lambda}, \Omega_{q}^{\mu}: \operatorname{Sin}_{q}(X) \rightarrow \operatorname{Sin}_{q-1}(X)$ be given by

$$
T_{q}^{\lambda}(\sigma)=\sigma \circ \tau_{q}^{\lambda}, \quad \Omega_{q}^{\mu}(\sigma)=\sigma \circ \omega_{q}^{\mu} .
$$

Then we have that 


$$
\begin{aligned}
& \left\{T_{q}^{\lambda} \mid \lambda \in M_{q}\right\} \subseteq\left\{\partial_{j} \circ \beta_{q}^{\nu} \mid(\nu, j) \in A_{q} \times\{0,1, \ldots, q\}\right\} \\
& \left\{\Omega_{q}^{\mu} \mid \mu \in N_{q}\right\} \subseteq\left\{\beta_{q-1}^{\alpha} \circ \partial_{j} \mid(j, \alpha) \in\{0,1, \ldots, q\} \times A_{q-1}\right\}
\end{aligned}
$$

and $\partial \beta_{q}=\sum_{\lambda \in M_{q}} t_{\lambda} T_{q}^{\lambda}, \beta_{q-1} \partial=\sum_{\mu \in N_{q}} s_{\mu} \Omega_{q}^{\mu}$. These results are also true for $q=1$ with some small, obvious changes in the notation (put $A_{0}=\{0\}, r_{0}=1, \beta_{0}^{0}=\mathrm{id}$ and $\left.\sigma_{0}^{0}=\mathrm{id}\right)$.

Define $\beta_{q}: \mathscr{C}_{q}(X) \rightarrow \mathscr{C}_{q}(X)$ by $\beta_{q}=\sum_{\nu \in A_{q}} r_{\nu}\left(\beta_{q}^{\nu}\right)_{*}$ for $q \geq 1$ and $\beta_{0}=$ id.

Lemma 4.1. $\beta: \mathscr{C}_{*}(X) \rightarrow \mathscr{C}_{*}(X)$ is a natural chain map.

PRoof. The map is natural by definition. For $q \geq 1$ we have

$$
\begin{aligned}
& \partial \beta_{q}=\sum_{j=0}^{q}(-1)^{j}\left(\partial_{j}\right)_{*}\left(\sum_{\nu \in A_{q}} r_{\nu}\left(\beta_{q}^{\nu}\right)_{*}\right)=\sum_{j=0}^{q} \sum_{\nu \in A_{q}}(-1)^{j} r_{\nu}\left(\partial_{j} \circ \beta_{q}^{\nu}\right)_{*} \\
& \beta_{q-1} \partial=\sum_{j=0}^{q} \sum_{\alpha \in A_{q-1}}(-1)^{j} r_{\alpha}\left(\beta_{q-1}^{\alpha} \circ \partial_{j}\right)_{*} \quad\left(\beta_{0} \partial=\sum_{j=0}^{1}(-1)^{j}\left(\partial_{j}\right)_{*}\right) .
\end{aligned}
$$

From the remarks before the lemma we conclude that

$$
\partial \beta_{q}=\sum_{\lambda \in M_{q}} t_{\lambda}\left(T_{q}^{\lambda}\right)_{*}, \quad \beta_{q-1} \partial=\sum_{\mu \in N_{q}} s_{\mu}\left(\Omega_{q}^{\mu}\right)_{*}
$$

which implies $\partial \beta_{q}=\beta_{q-1} \partial$.

In the following we write (4) as $s_{q}=\sum_{\alpha \in B_{q}} r_{\alpha} s_{q}^{\alpha}$ where $r_{\alpha} \in\{-1,1\}$ and $\left\{s_{q}^{\alpha} \mid \alpha \in B_{q}\right\}=M_{f}^{q} \cup M_{g}^{q}$. Here

$$
\begin{aligned}
& M_{f}^{q}=\left\{f_{q, \nu}^{k_{1} \ldots k_{q}} \mid \nu \in\{1, \ldots, q\},\left(k_{1}, \ldots, k_{q}\right) \in A_{q}\right\} \\
& M_{g}^{q}=\left\{g_{q, \nu}^{k_{\nu+1} \ldots k_{q}} \mid \nu \in\{1, \ldots, q-1\},\left(k_{\nu+1}, \ldots, k_{q}\right) \in A_{q}^{\nu+1}\right\} \cup\left\{h_{q, q}\right\}
\end{aligned}
$$

where $A_{q}^{p}$ is the set of tuples $\left(a_{p}, \ldots, a_{q}\right), a_{i}=0,1, \ldots, i, p=1,2, \ldots, q$. We then have that

$$
\partial s_{q}=\sum_{j=0}^{q+1} \sum_{\alpha \in B_{q}}(-1)^{j} r_{\alpha} \partial_{j} \circ s_{q}^{\alpha}, \quad s_{q-1} \partial=\sum_{j=0}^{q} \sum_{\gamma \in B_{q-1}}(-1)^{j} r_{\gamma} s_{q-1}^{\gamma} \circ \partial_{j}
$$

so $\partial s_{q}+s_{q-1} \partial=\beta_{q}-$ id is equivalent to

$$
\sum_{j=0}^{q+1} \sum_{\alpha \in B_{q}}(-1)^{j} r_{\alpha} \partial_{j} \circ s_{q}^{\alpha}+\sum_{j=0}^{q} \sum_{\gamma \in B_{q-1}}(-1)^{j} r_{\gamma} s_{q-1}^{\gamma} \circ \partial_{j}=\sum_{\nu \in A_{q}} r_{\nu} \beta_{q}^{\nu}-\mathrm{id} .
$$


Define $s_{q}: \mathscr{C}_{q}(X) \rightarrow \mathscr{C}_{q+1}(X)$ by $s_{q}=\sum_{\alpha \in B_{q}} r_{\alpha}\left(s_{q}^{\alpha}\right)_{*}$ for $q \geq 1$. For $q \leq 0$, $s_{q}=0$.

LEMMA 4.2. $s: \beta \simeq \operatorname{id}_{\mathscr{C}_{*}(X)}$ is a natural chain homotopy.

Proof. Naturality follows from the definition. We have $\beta_{q}-\mathrm{id}=$ $\sum_{\nu \in A_{q}} r_{\nu}\left(\beta_{q}^{\nu}\right)_{*}-\mathrm{id}$ and

$$
\partial s_{q}=\sum_{j=0}^{q+1} \sum_{\alpha \in B_{q}}(-1)^{j} r_{\alpha}\left(\partial_{j} \circ s_{q}^{\alpha}\right)_{*}, \quad s_{q-1} \partial=\sum_{j=0}^{q} \sum_{\gamma \in B_{q-1}}(-1)^{j} r_{\gamma}\left(s_{q-1}^{\gamma} \circ \partial_{j}\right)_{*} .
$$

Now evaluate (5) on $\imath_{q}$ and use the same procedure as in the remarks before lemma 4.1 to deduce that $s$ defines a chain homotopy $\partial s_{q}+s_{q-1} \partial=\beta_{q}-\mathrm{id}$.

Let $\mathscr{U}=\left\{U_{\alpha} \mid \alpha \in I\right\}$ be a family of subsets of $X$. We put $W_{\alpha}^{k}=\left(i_{\alpha}\right)_{\#}\left(\operatorname{Sin}_{k}\left(U_{\alpha}\right)\right)$ where $i_{\alpha}: U_{\alpha} \rightarrow X$ is the inclusion map. If $U_{\alpha}$ is open in $\mathrm{X}$ then $W_{\alpha}^{k}$ is open in $\operatorname{Sin}_{k}(X)$ and $\left(i_{\alpha}\right)_{\#}: \operatorname{Sin}_{k}\left(U_{\alpha}\right) \rightarrow W_{\alpha}^{k}$ is a homeomorphism.

Lemma 4.3. Let $n \in \mathrm{N}$. Then we have a natural chain homotopy $c: \beta^{n} \simeq \operatorname{id}_{\mathscr{C}_{*}(X)}$. If $\mu \in \mathscr{C}_{q}(X)$ and $\partial \mu \in \mathscr{C}_{q-1}^{\mathscr{U}}(X)$ then $c_{q-1}(\partial \mu) \in \mathscr{C}_{q}^{\mathscr{U}}(X)$.

Proof. Let $c_{q}=s_{q}\left(\mathrm{id}+\beta_{q}+\ldots+\beta_{q}^{n-1}\right): \mathscr{C}_{q}(X) \rightarrow \mathscr{C}_{q+1}(X)$. Then $c$ is a natural chain homotopy between $\beta^{n}$ and $\operatorname{id}_{\mathscr{C}_{*}(X)}$. Now let $q \geq 1$ and $\mu \in \mathscr{C}_{q}(X)$ and assume that $\partial \mu \in \mathscr{C}_{q-1}^{\mathscr{U}}(X)$. Write $\partial \mu=\sum_{j=1}^{n} r_{j} \mu_{j}, r_{j} \in \mathrm{R}$, $\mu_{j} \in \mathscr{C}_{q-1}^{U_{\alpha_{j}}}(X)$ and choose $\nu_{j} \in \mathscr{C}_{q-1}\left(U_{\alpha_{j}}\right)$ such that $\mu_{j}=i_{\alpha_{j}}\left(\nu_{j}\right)$. By naturality of $c$

$$
c_{q-1}(\partial \mu)=\sum_{j=1}^{n} r_{j} c_{q-1}\left(i_{\alpha_{j}}\left(\nu_{j}\right)\right)=\sum_{j=1}^{n} r_{j} i_{\alpha_{j}}\left(c_{q-1}\left(\nu_{j}\right)\right) \in \mathscr{C}_{q}^{\mathscr{U}}(X) .
$$

The main lemma is

LEMmA 4.4. Let $\mathscr{U}=\left\{U_{\alpha} \mid \alpha \in I\right\}$ be a family of subsets of $X$ such that $\operatorname{Int}(\mathscr{U})$ is a covering of $X$. Then for all $\mu \in \mathscr{C}_{q}(X)$ there exists a natural number $n$ so that the $n$ 'th iterate $\left(\beta_{q}\right)^{n}(\mu) \in \mathscr{C}_{q}^{\mathscr{U}}(X)$.

Proof. We may assume that $U_{\alpha}$ is open since $\mathscr{C}_{q}^{\operatorname{Int}(\mathscr{U})} \subseteq \mathscr{C}_{q}^{\mathscr{U}}(X)$. Now let $q \geq 1$. Since $\beta_{q}=\sum_{\nu \in A_{q}} r_{\nu}\left(\beta_{q}^{\nu}\right)_{*}$ we have

$$
\begin{aligned}
\left(\beta_{q}\right)^{k} & =\sum_{\nu_{1} \in A_{q}} \cdots \sum_{\nu_{k} \in A_{q}} r_{\nu_{1} \ldots \nu_{k}}\left(\beta_{q}^{\nu_{k}}\right)_{*} \circ \cdots \circ\left(\beta_{q}^{\nu_{1}}\right)_{*} \\
& =\sum_{\nu_{1} \in A_{q}} \cdots \sum_{\nu_{k} \in A_{q}} r_{\nu_{1} \ldots \nu_{k}}\left(\beta_{q}^{\nu_{k}} \circ \cdots \circ \beta_{q}^{\nu_{1}}\right)_{*}
\end{aligned}
$$


for every $k \in \mathrm{N}$ where $r_{\nu_{1} \ldots \nu_{k}}=\prod_{j=1}^{k} r_{\nu_{j}}$. From standard singular theory (cf. [D] (6.3) p. 41) we know that for given $\epsilon>0\left(\beta_{q}\right)^{k}\left(\imath_{q}\right)$ is a formal linear combination of simplices of diameter less than $\epsilon$ if $k$ is sufficiently large, say $k \geq n_{0}$. Now

$$
\left(\beta_{q}\right)^{k}\left(\imath_{q}\right)=\sum_{\nu_{1} \in A_{q}} \cdots \sum_{\nu_{k} \in A_{q}} r_{\nu_{1} \ldots \nu_{k}}\left(\beta_{q}^{\nu_{k}} \circ \cdots \circ \beta_{q}^{\nu_{1}}\right)\left(\imath_{q}\right)
$$

for every $k \in \mathrm{N}$ so $\operatorname{diam}\left(\beta_{q}^{\nu_{k}} \circ \cdots \circ \beta_{q}^{\nu_{1}}\left(\imath_{q}\right)\left(\Delta^{q}\right)\right)<\epsilon$ for all $k \geq n_{0}$ and all $\left(\nu_{1}, \ldots, \nu_{k}\right) \in\left(A_{q}\right)^{k}$. Here $\operatorname{diam}(C)$ denotes the diameter of $C$. For $\sigma \in \operatorname{Sin}_{q}(X), \mathscr{W}=\left\{\sigma^{-1}\left(U_{\alpha}\right) \mid \alpha \in I\right\}$ is an open covering of $\Delta^{q}$. This being compact there exists an $\epsilon_{\sigma}>0$ such that for $C \subseteq \Delta^{q}$ of diameter less than $\epsilon_{\sigma}$, there exist an index $\alpha$ with $C \in \sigma^{-1}\left(U_{\alpha}\right)\left(\epsilon_{\sigma}\right.$ is the Lebesgue number of the covering $\mathscr{W})$. Choose $n_{\sigma}$ such that for all $k \geq n_{\sigma}$ and all $\left(\nu_{1}, \ldots, \nu_{k}\right) \in\left(A_{q}\right)^{k}$ we have the implications

$$
\begin{aligned}
& \operatorname{diam}\left(\beta_{q}^{\nu_{k}} \circ \cdots \circ \beta_{q}^{\nu_{1}}\left(\imath_{q}\right)\left(\Delta^{q}\right)\right)<\epsilon_{\sigma} \Rightarrow \exists \alpha \in I: \beta_{q}^{\nu_{k}} \circ \cdots \circ \beta_{q}^{\nu_{1}}\left(\imath_{q}\right)\left(\Delta^{q}\right) \subseteq \sigma^{-1}\left(U_{\alpha}\right) \\
& \Rightarrow \exists \alpha \in I: \beta_{q}^{\nu_{k}} \cdots \cdots \circ \beta_{q}^{\nu_{1}}(\sigma) \in W_{\alpha}^{q} .
\end{aligned}
$$

Now let $k \geq n_{\sigma}$ and $\left(\nu_{1}, \ldots, \nu_{k}\right) \in\left(A_{q}\right)^{k}$ and choose $\alpha \in I$ such that $\beta_{q}^{\nu_{k}} \circ \cdots \circ \beta_{q}^{\nu_{1}}(\sigma) \in W_{\alpha}^{q}$. Since $W_{\alpha}^{q}$ is an open subset of $\operatorname{Sin}_{q}(X)$ and $\beta_{q}^{\nu_{k}} \circ \cdots \circ \beta_{q}^{\nu_{1}}: \operatorname{Sin}_{q}(X) \rightarrow \operatorname{Sin}_{q}(X)$ is continuous, it follows that there is an open neighborhood $U_{\sigma}^{\nu_{1} \ldots \nu_{k}}$ of $\sigma$ in $\operatorname{Sin}_{q}(X)$ such that $\beta_{q}^{\nu_{k}} \circ \cdots \circ$ $\beta_{q}^{\nu_{1}}\left(U_{\sigma}^{\nu_{1} \ldots \nu_{k}}\right) \subseteq W_{\alpha}^{q}$. Set $U_{\sigma}^{k}=\bigcap_{\nu_{1} \in A_{q}} \cdots \bigcap_{\nu_{k} \in A_{q}} U_{\sigma}^{\nu_{1} \ldots \nu_{k}}$. It is an open neighboorhood of $\sigma$ in $\operatorname{Sin}_{q}(X)$ and for all $\left(\nu_{1}, \ldots, \nu_{k}\right) \in\left(A_{q}\right)^{k}$ there is an index $\alpha$ with $\beta_{q}^{\nu_{k}} \circ \cdots \circ \beta_{q}^{\nu_{1}}\left(U_{\sigma}^{k}\right) \subseteq W_{\alpha}^{q}$. Let $\mu \in \mathscr{C}_{q}(X)$ be a chain with $\operatorname{Supp}(\mu) \subseteq K$ where $K \subseteq \operatorname{Sin}_{q}(X)$ is compact and set $O_{\sigma}=U_{\sigma}^{n_{\sigma}}$. Since $\left\{O_{\sigma}\right\}_{\sigma \in \operatorname{Sin}_{q}(X)}$ is a covering of $K$ with open subsets of $\operatorname{Sin}_{q}(X)$ we can find $\sigma_{1}, \ldots, \sigma_{l} \in \operatorname{Sin}_{q}(X)$ such that $K \subseteq O_{\sigma_{1}} \cup \ldots \cup O_{\sigma_{l}}$. Set $n_{j}=n_{\sigma_{j}}$ for $j \in\{1, \ldots, l\}$ and set $n=\max \left\{n_{1}, \ldots, n_{l}\right\}$ and let $\tau \in K$. Choose a $j \in\{1, \ldots, l\}$ such that $\tau \in O_{\sigma_{j}}$. Then for all $\left(\nu_{1}, \ldots, \nu_{n_{j}}\right) \in\left(A_{q}\right)^{n_{j}}$ there is an index $\alpha$ with $\beta_{q}^{\nu_{n_{j}}} \circ \cdots \circ \beta_{q}^{\nu_{1}}(\tau) \in W_{\alpha}^{q}$. Now let $k \geq n_{j}$ and $\left(\nu_{1}, \ldots, \nu_{k}\right) \in\left(A_{q}\right)^{k}$. Choose $\alpha \in I$ such that $\beta_{q}^{\nu_{n_{j}}} \circ \cdots \circ \beta_{q}^{\nu_{1}}(\tau) \in W_{\alpha}^{q}$. Then $\beta_{q}^{\nu_{k}} \circ \cdots \circ \beta_{q}^{\nu_{1}}(\tau) \in W_{\alpha}^{q}$. (Let $\omega \in W_{\alpha}^{q}$. Then $\omega\left(\Delta^{q}\right) \subseteq U_{\alpha}$. We therefore have

$$
\beta_{q}^{\nu}(\omega)\left(\Delta^{q}\right)=\omega \circ \sigma_{q}^{\nu}\left(\Delta^{q}\right) \subseteq \omega\left(\Delta^{q}\right) \subseteq U_{\alpha}
$$

for $\nu \in A_{q}$ so $\beta_{q}^{\nu}(\omega) \in W_{\alpha}^{q}$.) Thus to each $\left(\nu_{1}, \ldots, \nu_{n}\right) \in\left(A_{q}\right)^{n}$ and $\tau \in K$ we can find an index $\alpha$ with $\beta_{q}^{\nu_{n}} \circ \cdots \circ \beta_{q}^{\nu_{1}}(\tau) \in W_{\alpha}^{q}$. Since $\beta_{q}^{\nu_{n}} \circ \cdots \circ \beta_{q}^{\nu_{1}}$ is continuous $L=\beta_{q}^{\nu_{n}} \circ \cdots \circ \beta_{q}^{\nu_{1}}(K)$ is a compact subset of $\operatorname{Sin}_{q}(X)$; actually $L \subseteq W^{q}=\bigcup_{\alpha \in I} W_{\alpha}^{q}$. The support of $\lambda=\left(\beta_{q}^{\nu_{n}} \circ \cdots \circ \beta_{q}^{\nu_{1}}\right)_{*}(\mu)$ is contained in $L$. Choose $\alpha_{1}, \ldots, \alpha_{m} \in I$ such that $L \subseteq W_{\alpha_{1}}^{q} \cup \ldots \cup W_{\alpha_{m}}^{q}$ and let $V_{j}=W_{\alpha_{j}}^{q} \cap L$. Since $V_{j} \in \mathscr{B}\left(\operatorname{Sin}_{q}(X)\right)$ it follows that $\mathscr{B}\left(V_{j}\right) \subseteq \mathscr{B}\left(\operatorname{Sin}_{q}(X)\right)$. 
The restriction $\lambda_{j}$ of $\lambda$ to $\mathscr{B}\left(V_{j}\right)$ defines a real Borel measure on $V_{j}$, $j=1,2, \ldots, m .\left\{V_{j}\right\}_{j=1}^{m}$ is an open covering of $L$ and $L$ is normal since it is a closed subset of the normal space $\operatorname{Sin}_{q}(X)$. We can therefore choose a partition of unity $\left\{\rho_{j}\right\}_{j=1}^{m}$ subordinated to the covering $\left\{V_{j}\right\}_{j=1}^{m}$. The maps $\rho_{j}: V_{j} \rightarrow \mathrm{R}$ are continuous and therefore Borel measurable. Now let

$$
\eta_{j}(B)=\int_{B} \rho_{j} d\left(\lambda_{j}\right), \quad B \in \mathscr{B}\left(V_{j}\right), \quad j=1,2, \ldots, m .
$$

Since $\rho_{j}(L) \subseteq[0,1]$ it follows that $\rho_{j} \in L^{1}\left(\lambda_{j}\right)$. This implies that $\eta_{j}$ is a signed Borel measure on $V_{j}$ of bounded total variation, and we can define $\mu_{j}$ by

$$
\mu_{j}(B)=\eta_{j}\left(\left(i_{\alpha_{j}}\right)_{\#}(B) \cap L\right), \quad B \in \mathscr{B}\left(\operatorname{Sin}_{q}\left(U_{\alpha_{j}}\right)\right), j=1,2, \ldots, m .
$$

Since $V_{j} \in \mathscr{B}\left(W_{\alpha_{j}}^{q}\right)$ we have

$$
\mathscr{B}\left(V_{j}\right)=\left\{B \cap V_{j} \mid B \in \mathscr{B}\left(W_{\alpha_{j}}^{q}\right)\right\}=\left\{B \cap L \mid B \in \mathscr{B}\left(W_{\alpha_{j}}^{q}\right)\right\} .
$$

Now $\left(i_{\alpha_{j}}\right)_{\#}: \operatorname{Sin}_{q}\left(U_{\alpha_{j}}\right) \rightarrow W_{\alpha_{j}}^{q}$ is a homeomorphism, so induces a bijection $\mathscr{P}\left(\left(i_{\alpha_{j}}\right)_{\#}\right): \mathscr{B}\left(\operatorname{Sin}_{q}\left(U_{\alpha_{j}}\right)\right) \rightarrow \mathscr{B}\left(W_{\alpha_{j}}^{q}\right)$. Thus $\mu_{j}$ is a well defined real valued Borel measure on $\operatorname{Sin}_{q}\left(U_{\alpha_{j}}\right)$. If we put $L_{j}=\operatorname{Supp}_{L}\left(\rho_{j}\right) \subseteq V_{j} \subseteq W_{\alpha_{j}}^{q}$ then

$$
\begin{aligned}
\eta_{j}(B) & =\int_{B} \rho_{j} d\left(\lambda_{j}\right)=\int_{V_{j}} \chi_{B} \rho_{j} d\left(\lambda_{j}\right)=\int_{V_{j}} \chi_{B} \chi_{L_{j}} \rho_{j} d\left(\lambda_{j}\right) \\
& =\int_{V_{j}} \chi_{B \cap L_{j}} \rho_{j} d\left(\lambda_{j}\right)=\int_{B \cap L_{j}} \rho_{j} d\left(\lambda_{j}\right)=\eta_{j}\left(B \cap L_{j}\right)
\end{aligned}
$$

for $B \in \mathscr{B}\left(V_{j}\right)$, where $\chi_{B}$ is the characteristic function of $B$ etc. Observe here that $L_{j}$ is a closed subset of $L$ hence of $\operatorname{Sin}_{q}(X)$, so $L_{j} \in \mathscr{B}\left(\operatorname{Sin}_{q}(X)\right)$. Moreover $B \in \mathscr{B}\left(V_{j}\right) \subseteq \mathscr{B}\left(\operatorname{Sin}_{q}(X)\right) \quad$ so $\quad B \cap L_{j} \subseteq \mathscr{B}\left(\operatorname{Sin}_{q}(X)\right)$. But then $B \cap L_{j} \subseteq \mathscr{B}\left(V_{j}\right)$ since $B \cap L_{j} \subseteq V_{j} \in \mathscr{B}\left(\operatorname{Sin}_{q}(X)\right)$. This shows that the above calculations are allowed. Also $M_{j}=\left(i_{\alpha_{j}}\right)_{\#}^{-1}\left(L_{j}\right)$ is a compact subset of $\operatorname{Sin}_{q}\left(U_{\alpha_{j}}\right)$ and we have

$$
\begin{aligned}
\mu_{j}(D) & =\eta_{j}\left(\left(i_{\alpha_{j}}\right)_{\#}(D) \cap L\right)=\eta_{j}\left(\left(i_{\alpha_{j}}\right)_{\#}(D) \cap L \cap L_{j}\right) \\
& =\eta_{j}\left(\left(i_{\alpha_{j}}\right)_{\#}(D) \cap L \cap\left(i_{\alpha_{j}}\right)_{\#}\left(M_{j}\right)\right) \\
& =\eta_{j}\left(\left(i_{\alpha_{j}}\right)_{\#}\left(D \cap M_{j}\right) \cap L\right)=\mu_{j}\left(D \cap M_{j}\right)
\end{aligned}
$$

for all $D \in \mathscr{B}\left(\operatorname{Sin}_{q}\left(U_{\alpha_{j}}\right)\right)$, i.e. $\mu_{j} \in \mathscr{C}_{q}\left(U_{\alpha_{j}}\right)$. If $B \in \mathscr{B}\left(\operatorname{Sin}_{q}(X)\right)$ we have 


$$
\begin{aligned}
\sum_{j=1}^{m} i_{\alpha_{j}}\left(\mu_{j}\right)(B) & =\sum_{j=1}^{m} \mu_{j}\left(\left(i_{\alpha_{j}}\right)_{\#}^{-1}\left(B \cap W_{\alpha_{j}}^{q}\right)\right)=\sum_{j=1}^{m} \eta_{j}\left(B \cap W_{\alpha_{j}}^{q} \cap L\right) \\
& =\sum_{j=1}^{m} \int_{B \cap V_{j}} \rho_{j} d\left(\lambda_{j}\right)=\sum_{j=1}^{m} \int_{V_{j}} \chi_{B \cap V_{j}} \rho_{j} d\left(\lambda_{j}\right) \\
& =\sum_{j=1}^{m} \int_{\operatorname{Sin}_{q}(X)} \chi_{B \cap V_{j}} \xi_{j} d \lambda,
\end{aligned}
$$

where $\xi_{j}: \operatorname{Sin}_{q}(X) \rightarrow \mathrm{R}$ is the Borel function defined by $\xi_{j}(x)=\rho_{j} \chi_{L}(x)$, $x \in \operatorname{Sin}_{q}(X)$. Now $\chi_{L \cap B}=\chi_{B \cap V_{j}}+\chi_{(L \cap B) \backslash V_{j}}$ and $\xi_{j}=0$ on $(L \cap B) \backslash V_{j}$ so $\chi_{B \cap V_{j}} \xi_{j}=\chi_{L \cap B} \xi_{j}$. Since $\sum \xi_{j} \equiv 1$ on $L$,

$$
\begin{aligned}
\sum_{j=1}^{m} i_{\alpha_{j}}\left(\mu_{j}\right)(B) & =\sum_{j=1}^{m} \int_{\operatorname{Sin}_{q}(X)} \chi_{B \cap L} \xi_{j} d(\lambda)=\int_{\operatorname{Sin}_{q}(X)} \chi_{B \cap L} d(\lambda) \\
& =\lambda(B \cap L)=\lambda(B) .
\end{aligned}
$$

It follows that $\lambda=\sum_{j=1}^{m} i_{\alpha_{j}}\left(\mu_{j}\right)$ and we conclude that

$$
\left(\beta_{q}\right)^{n}(\mu)=\sum_{\nu_{1} \in A_{q}} \cdots \sum_{\nu_{n} \in A_{q}} r_{\nu_{1} \ldots \nu_{n}}\left(\beta_{q}^{\nu_{n}} \circ \cdots \circ \beta_{q}^{\nu_{1}}\right)_{*}(\mu) \in \mathscr{C}_{q}^{\mathscr{U}}(X) .
$$

For $q=0$ the result easily follows by the preceding. Observe that $W^{0}=\bigcup_{\alpha \in I} W_{\alpha}^{0}=\operatorname{Sin}_{0}(X)$ and skip the first part of the proof and let $\lambda=\mu$ and $L=K$ in the last part the proof.

Proof of theorem 3.1. Set $\mathscr{C}_{*}(X ; \mathscr{U})=\mathscr{C}_{*}(X) / \mathscr{C}_{*}^{\mathscr{U}}(X)$, giving the exact sequence

$$
0 \longrightarrow \mathscr{C}_{*}^{\mathscr{U}}(X) \stackrel{i}{\longrightarrow} \mathscr{C}_{*}(X) \stackrel{\pi}{\longrightarrow} \mathscr{C}_{*}(X ; \mathscr{U}) \longrightarrow 0 .
$$

We must show that $H_{*}\left(\mathscr{C}_{*}(X ; \mathscr{U})\right)=0$. Let $\mu+\mathscr{C}_{q}^{\mathscr{U}}(X) \in Z_{q}\left(\mathscr{C}_{*}(X ; \mathscr{U})\right)$. Then

$$
\bar{\partial}\left(\mu+\mathscr{C}_{q}^{\mathscr{U}}(X)\right)=\partial \mu+\mathscr{C}_{q-1}^{\mathscr{U}}(X)=0+\mathscr{C}_{q-1}^{\mathscr{U}}(X) \Leftrightarrow \partial \mu \in \mathscr{C}_{q-1}^{\mathscr{U}}(X) .
$$

By lemma 4.4 we can choose $n \in \mathrm{N}$ such that $\left(\beta_{q}\right)^{n}(\mu) \in \mathscr{C}_{q}^{\mathscr{U l}}(X)$. Let $c$ be the chain homotopy between $\beta^{n}$ and $i d_{\mathscr{C}_{*}(X)}$, see lemma 4.3. Set $y=\left(\beta_{q}\right)^{n}(\mu)-c_{q-1}(\partial \mu)$ and $x=-c_{q}(\mu)$. Since $\partial \mu \in \mathscr{C}_{q-1}^{\mathscr{I}}(X)$ it follows from lemma 4.3 that $c_{q-1}(\partial \mu) \in \mathscr{C}_{q}^{\mathscr{I I}}(X)$ which implies that $y \in \mathscr{C}_{q}^{\mathscr{I I}}(X)$. Moreover we have that $x \in \mathscr{C}_{q+1}(X)$ and $\mu=i d(\mu)=\left(\beta_{q}\right)^{n}(\mu)-c_{q-1}(\partial \mu)-$ $\partial\left(c_{q}(\mu)\right)=y+\partial x$. This implies that

$$
\mu-\partial x \in \mathscr{C}_{q}^{\mathscr{U}}(X) \Leftrightarrow \mu+\mathscr{C}_{q}^{\mathscr{U}}(X)=\partial x+\mathscr{C}_{q}^{\mathscr{U}}(X)=\bar{\partial}\left(x+\mathscr{C}_{q+1}^{\mathscr{U}}(X)\right) .
$$

But then $\mu+\mathscr{C}_{q}^{\mathscr{U}}(X) \in B_{q}\left(\mathscr{C}_{*}(X ; \mathscr{U})\right)$. 


\section{Measure homology and $\mathrm{CW}$-complexes.}

In this section we show that the measure homology groups are isomorphic to the singular homology groups on the category of CW-complexes. The problem is that not all $\mathrm{CW}$-complexes are metrizable so we need the following result. Let $X$ be a topological space and let $\left\{X_{\alpha}\right\}_{\alpha \in I}$ be the family of compact subsets of $X$ partially ordered by inclusion. Let $i_{\alpha}^{\beta}: X_{\alpha} \rightarrow X_{\beta}$ be the inclusion when $X_{\alpha} \subseteq X_{\beta}$. Then $\left\{H_{*}^{\mu}\left(X_{\alpha}\right)\right\}_{\alpha \in I}$ forms a direct system of real vector spaces with the linear maps $f_{\alpha}^{\beta}=H_{*}^{\mu}\left(i_{\alpha} \beta\right): H_{*}^{\mu}\left(X_{\alpha}\right) \rightarrow H_{*}^{\mu}\left(X_{\beta}\right)$ induced by the inclusion maps. We now have

Proposition 5.1.

$$
H_{*}^{\mu}(X) \cong \underset{\alpha}{\lim } H_{*}^{\mu}\left(X_{\alpha}\right)
$$

Proof. Let $i_{\alpha}: X_{\alpha} \rightarrow X$ be the inclusion maps, $f_{\alpha}=H_{*}^{\mu}\left(i_{\alpha}\right): H_{*}^{\mu}\left(X_{\alpha}\right) \rightarrow$ $H_{*}^{\mu}(X)$ and let $f=\bigoplus_{\alpha \in I} f_{\alpha}: \bigoplus_{\alpha \in I} H_{*}^{\mu}\left(X_{\alpha}\right) \rightarrow H_{*}^{\mu}(X)$. If $\sum_{i=1}^{n} x_{\alpha_{i}} \in \bigoplus_{\alpha \in I}$ $H_{*}^{\mu}\left(X_{\alpha}\right)$ and $\beta \in I$ is such that $X_{\alpha_{i}} \subseteq X_{\beta}$ for $i=1,2, \ldots, n$ and $\sum_{i=1}^{n} f_{\alpha_{i}}^{\beta}\left(x_{\alpha_{i}}\right)=0$, then $0=f_{\beta}\left(\sum_{i=1}^{n} f_{\alpha_{i}}^{\beta}\left(x_{\alpha_{i}}\right)\right)=\sum_{i=1}^{n} f_{\alpha_{i}}\left(x_{\alpha_{i}}\right)=f\left(\sum_{i=1}^{n} x_{\alpha_{i}}\right)$. Thus $f$ induces a linear map

$$
\tilde{f}: \underset{\alpha}{\lim } H_{*}^{\mu}\left(X_{\alpha}\right) \rightarrow H_{*}^{\mu}(X) .
$$

Now let $[\mu] \in H_{q}^{\mu}(X)$ and choose a compact subset $K \subseteq \operatorname{Sin}_{q}(X)$ with $\operatorname{Supp}(\mu) \subseteq K$. The evaluation map $\omega: \operatorname{Sin}_{q}(X) \times \Delta^{q} \rightarrow X$ defined by $\omega(\sigma, x)=\sigma(x)$ is continuous since $\Delta^{q}$ is compact so $A=\omega\left(K \times \Delta^{q}\right)$ is a compact subset of $X$. Now if $\sigma \in K$ we have that $\sigma\left(\Delta^{q}\right) \subseteq A$, and it follows that $K \subseteq j_{\#}\left(\operatorname{Sin}_{q}(A)\right)$ where $j: A \rightarrow X$ is the inclusion. But then $L=j_{\#}^{-1}(K)$ is a compact subset of $\operatorname{Sin}_{q}(A)$. The homeomorphism $j_{\#}: L \rightarrow K$ induces a bijection $Q=\mathscr{P}\left(j_{\#}\right): \mathscr{B}(L) \rightarrow \mathscr{B}(K)$. Since $K \in \mathscr{B}\left(\operatorname{Sin}_{q}(X)\right)$ we have that $\mathscr{B}(K) \subseteq \mathscr{B}\left(\operatorname{Sin}_{q}(X)\right)$. Moreover $\mathscr{B}(L)=\left\{L \cap D \mid D \in \mathscr{B}\left(\operatorname{Sin}_{q}(A)\right)\right\}$, so we can define a signed Borel measure $\nu$ on $\operatorname{Sin}_{q}(A)$ of bounded total variation by $\nu(D)=\mu \circ Q(L \cap D)$. By definition $\operatorname{Supp}(\nu) \subseteq L \quad$ so $\quad \nu \in \mathscr{C}_{q}(A)$. If $B \in \mathscr{B}\left(\operatorname{Sin}_{q}(X)\right)$ we have

$$
\begin{aligned}
j(\nu)(B) & =\nu\left(j_{\#}^{-1}(B)\right)=\mu \circ Q\left(L \cap j_{\#}^{-1}(B)\right)=\mu \circ Q\left(j_{\#}^{-1}(K \cap B)\right) \\
& =\mu(K \cap B)=\mu(B) .
\end{aligned}
$$

Now $0=\partial \mu=j(\partial \nu)$ and by lemma $2.1 j: \mathscr{C}_{*}(A) \rightarrow \mathscr{C}_{*}(X)$ is injective so $\partial \nu=0$. All in all we see that $[\mu]=H_{q}^{\mu}(j)([\nu])$ so $f$ and thus $\tilde{f}$ is surjective.

Suppose that $\sum_{i=1}^{n}\left[\mu_{\alpha_{i}}\right] \in \bigoplus_{\alpha \in I} H_{*}^{\mu}\left(X_{\alpha}\right)$ with $f\left(\sum_{i=1}^{n}\left[\mu_{\alpha_{i}}\right]\right)=0$. Since 


$$
f\left(\sum_{i=1}^{n}\left[\mu_{\alpha_{i}}\right]\right)=\sum_{i=1}^{n} f_{\alpha_{i}}\left(\left[\mu_{\alpha_{i}}\right]\right)=\sum_{i=1}^{n}\left[i_{\alpha_{i}}\left(\mu_{\alpha_{i}}\right)\right]=\left[\sum_{i=1}^{n} i_{\alpha_{i}}\left(\mu_{\alpha_{i}}\right)\right]
$$

we can choose $\nu \in \mathscr{C}_{q+1}(X)$ with $\partial \nu=\sum_{i=1}^{n} i_{\alpha_{i}}\left(\mu_{\alpha_{i}}\right)$. Let $K \subseteq \operatorname{Sin}_{q+1}(X)$ be a compact support of $\nu$ and let $A=\omega\left(K \times \Delta^{q+1}\right)$ where $\omega: \operatorname{Sin}_{q+1}(X) \times \Delta^{q+1} \rightarrow X$ is the continuous evaluation map. Then $X_{\beta}=A \cup \bigcup_{i=1}^{n} X_{\alpha_{i}}$ is a compact subset of $X$. For $\sigma \in K, \sigma\left(\Delta^{q+1}\right) \subseteq A \subseteq X_{\beta}$, so we can choose $\lambda \in \mathscr{C}_{q+1}\left(X_{\beta}\right)$ with $i_{\beta}(\lambda)=\nu$. Since

$$
i_{\beta}(\partial \lambda)=\partial i_{\beta}(\lambda)=\partial \nu=\sum_{i=1}^{n} i_{\alpha_{i}}\left(\mu_{\alpha_{i}}\right)=i_{\beta}\left(\sum_{i=1}^{n} i_{\alpha_{i}}^{\beta}\left(\mu_{\alpha_{i}}\right)\right)
$$

and since $i_{\beta}: \mathscr{C}_{q}\left(X_{\beta}\right) \rightarrow \mathscr{C}_{q}(X)$ is injective, $\sum_{i=1}^{n} i_{\alpha_{i}}^{\beta}\left(\mu_{\alpha_{i}}\right)$ is a boundary in $\mathscr{C}_{q}\left(X_{\beta}\right)$. Hence $\sum_{i=1}^{n} f_{\alpha_{i}}^{\beta}\left(\left[\mu_{\alpha_{i}}\right]\right)=\sum_{i=1}^{n}\left[i_{\alpha_{i}}^{\beta}\left(\mu_{\alpha_{i}}\right)\right]=0$.

COROLlaRY 5.2. The measure homology groups and the singular homology groups are isomorphic on the category of $C W$-complexes.

Proof. This follows by the fact that a compact subset of a CW-complex is metrizable.

\section{REFERENCES}

[D] A. Dold, Lectures on Algebraic Topology, New York: Springer 1972.

[S] A. H. Stone, A note on paracompactness and normality of mapping spaces, Proc. Amer. Math. Soc. 14 (1963), 81-83.

[T] W. Thurston, The Geometry and Topology of 3-manifolds, Princeton University, Lecture notes, 1978.

MATEMATISK INSTITUT AARHUS UNIVERSITET BYGNING 530 8000 AARHUS C DENMARK

email: kold@mi.aau.dk 